Check for updates

Cite this: Phys. Chem. Chem. Phys., 2019, 21, 25362

Received 29th August 2019,

Accepted 30th September 2019

DOI: $10.1039 / \mathrm{c} 9 \mathrm{cp} 04790 \mathrm{e}$

rsc.li/pccp

\section{Considerable matrix shift in the electronic transitions of helium-solvated cesium dimer cation $\mathrm{Cs}_{2} \mathrm{He}_{n}^{+} \dagger$}

\author{
Lorenz Kranabetter, (D) a Nina K. Bersenkowitsch, ${ }^{a}$ Paul Martini, ${ }^{a}$ \\ Michael Gatchell, (D) ab Martin Kuhn, ${ }^{a}$ Felix Laimer, (D) ${ }^{a}$ Arne Schiller, ${ }^{a}$ \\ Martin K. Beyer, (D) Milan Ončák (DD *a and Paul Scheier (DD *a
}

\begin{abstract}
We investigate the photodissociation of helium-solvated cesium dimer cations using action spectroscopy and quantum chemical calculations. The spectrum of $\mathrm{Cs}_{2} \mathrm{He}^{+}$shows three distinct absorption bands into both bound and dissociative states. Upon solvation with further helium atoms, considerable shifts of the absorption bands are observed, exceeding $0.1 \mathrm{eV}\left(850 \mathrm{~cm}^{-1}\right)$ already for $\mathrm{Cs}_{2} \mathrm{He}_{10}{ }^{+}$, along with significant broadening. The shifts are highly sensitive to the character of the excited state. Our calculations show that helium atoms adsorb on the ends of $\mathrm{Cs}_{2}{ }^{+}$. The shifts are particularly pronounced if the excited state orbitals extend to the area occupied by the helium atoms. In this case, Pauli repulsion leads to a deformation of the excited state orbitals, resulting in the observed blue shift of the transition. Since the position of the weakly bound helium atoms is ill defined, Pauli repulsion also explains the broadening.
\end{abstract}

\section{Introduction}

Vibrationally resolved electronic spectroscopy in combination with theoretical calculations is a powerful technique to determine details of the geometrical arrangement and electronic structure of molecules or clusters. The quality of vibrationally resolved spectra depends critically on the temperature, as recently demonstrated for the assignment of the structure of $\mathrm{Au}_{4}{ }^{+}{ }^{1}$ This has triggered the development of several cryogenic techniques, i.e., molecular beams ${ }^{2,3}$ matrix isolation, ${ }^{4,5}$ helium droplets, ${ }^{6,7}$ cryogenic traps ${ }^{8-20}$ and cryogenic storage rings. ${ }^{21-25}$ The low density of ionic targets requires special techniques, such as cavity ring down ${ }^{26}$ or action spectroscopy. ${ }^{27,28}$ Tagging of ions with a weakly bound messenger turns out to be particularly suitable to measure absorption lines of ions, albeit leading to a matrix shift. ${ }^{10,29}$ The binding energy of helium to ions is lower than for any other atom or molecule, leading to a minimum matrix shift. ${ }^{30}$

Whereas only very few He atoms can be attached to singlycharged ions in cold traps, ions can be solvated with almost any number of $\mathrm{He}$ atoms when formed in doped helium nanodroplets. In the case of $\mathrm{C}_{60}{ }^{+}$, a remarkable linear red-shift of the

\footnotetext{
${ }^{a}$ Institut für Ionenphysik und Angewandte Physik, Universität Innsbruck, Technikerstr. 25, A-6020 Innsbruck, Austria. E-mail: Milan.Oncak@uibk.ac.at, Paul.Scheier@uibk.ac.at

${ }^{b}$ Department of Physics, Stockholm University, 10691 Stockholm, Sweden

$\dagger$ Electronic supplementary information (ESI) available: Relative stabilities of calculated isomers, method benchmarks, spin-orbit couplings, complete experimental spectra, Cartesian coordinates. See DOI: 10.1039/c9cp04790e
}

absorption lines for the first $32 \mathrm{He}$ atoms attached could be used to extrapolate to the absorption line of the bare ion. ${ }^{31-33}$ In combination with theory, the experimental results of the matrix shift as well as the width of the absorption lines provide unprecedented insight into the details of the solvation of ions with helium, including phase transitions and isomeric effects.

Alkali metal atoms reside on dimples at the surface of helium droplets, as shown in numerous experimental ${ }^{21,34-40}$ and theoretical studies. ${ }^{41-46}$ Alkali metal clusters, however, are submerged into the cluster above a critical cluster size. ${ }^{46-48}$ The groups of Ernst and Stienkemeier investigated photoionization of Cs atoms and the submersion of $\mathrm{Cs}^{+}$into He droplets. ${ }^{34,39}$ Chen et al. managed to solvate $\mathrm{Cs}^{+}$ions with several million helium atoms via pickup of $\mathrm{Cs}^{+}$formed upon thermionic emission into large He droplets. ${ }^{49}$ Dopant ions ejected from charged helium droplets are sometimes complexed with a few helium atoms when recorded via mass spectrometry. The first solvation layer is particularly strongly bound, and its closure is typically reflected as a clear intensity drop of the ion series $\mathrm{MHe}_{n}^{+}$(with $\mathrm{M}^{+}$the alkali metal ion) in mass spectra. ${ }^{48,50-52}$ Path integral or basin-hopping methods reproduce the experiments very well. ${ }^{44,45}$ It was also shown previously that helium adsorption might lead to appreciable spectral shifts. ${ }^{53}$

Spectroscopic properties of the $\mathrm{Cs}_{2}{ }^{+}$ion were investigated previously in experimental ${ }^{54,55}$ and theoretical studies ${ }^{56-58}$ within the series of alkali metal dimers, assigning the lowest electronically excited states. In the present study, we explore photodissociation of $\mathrm{Cs}_{2} \mathrm{He}_{n}^{+}$via action spectroscopy to assess helium 
solvation effects in a seemingly simple diatomic system. Singlereference and multi-reference $a b$ initio calculations including spin-orbit coupling reproduce the experimental results and provide an assignment of the electronic transitions. A pronounced blue-shift of more than $0.1 \mathrm{eV}\left(850 \mathrm{~cm}^{-1}\right)$ for absorption into the $1^{2} \Sigma_{\mathrm{u}}^{+}$state for $\mathrm{Cs}_{2} \mathrm{He}_{n}^{+}$with $n=10$ provides clear evidence that the He atoms preferentially occupy positions along the axis of the cesium dimer cation.

\section{Methods}

$\mathrm{Cs}_{m} \mathrm{He}_{n}^{+}$ions are formed upon electron irradiation $(85 \mathrm{eV}, 260 \mu \mathrm{A})$ of Cs doped He nanodroplets (average size about $10^{5}$ atoms, 2.7 $\mathrm{MPa}, 9.95 \mathrm{~K}$ expansion conditions). Penning ionization via electronically excited $\mathrm{He}^{*}$ will be the dominant ionization channel for the heliophobic cesium atoms. ${ }^{7,59-61}$ Ionized cesium atoms and clusters have a substantially stronger interaction with the $\mathrm{He}$ matrix than their corresponding neutral precursors, thus leading to their submersion into the droplet. ${ }^{46}$ Low-mass ions ejected from the large multiply charged droplet are deflected by 90 degrees relative to the neutral beam via electro-static lenses. The beam is guided into the extraction region of a high-resolution time of flight mass spectrometer, where it is merged with a laser beam from a pulsed tuneable light source (EKSPLA NT 242, 210-2600 nm). Every tenth extraction pulse of the mass spectrometer operated at $10 \mathrm{kHz}$ is irradiated with the laser (repetition rate $1 \mathrm{kHz}$ ), thereby enabling simultaneous measurement of mass spectra with and without laser light. Upon photon absorption, all adsorbed He atoms are typically lost from $\mathrm{Cs}_{m} \mathrm{He}_{n}^{+}$, and an optical photodissociation spectrum is derived from the depletion signal. A detailed description of the experiment can be found in the ESI $\dagger$ (Fig. S9) and elsewhere. ${ }^{62}$

To obtain a quantum chemical description of the optical spectra, ground state structures of $\mathrm{Cs}_{2} \mathrm{He}_{n}^{+}$ions were first modelled at the coupled cluster singles doubles (CCSD) level with the def2QZVP basis set on Cs and def2TZVP on He. The position of the weakly bound $\mathrm{He}$ atoms, residing in shallow potential wells, is very sensitive to the quality of the basis set used on Cs. With the triple-zeta quality basis set def2TZVP used on Cs, a bent structure is predicted for $\mathrm{Cs}_{2} \mathrm{He}^{+}$; a linear $\mathrm{Cs}_{2} \mathrm{He}^{+}$ structure is obtained with the def2QZVP basis set on Cs and def2TZVP on He, and this does not change when the def2QZVPPD basis set is used on both Cs and He. To validate the theory as much as possible, excited states were modelled on various theory levels: time-dependent density functional theory (TDDFT) with the CAM-B3LYP functional; equation of motion - CCSD (EOMCCSD); multi-reference configuration interaction (MRCI) with an active space of one electron in 5 or 17 orbitals, $\operatorname{MRCI}(1,5)$ and MRCI(1,17), respectively; def2QZVPPD basis set was used on all atoms for excited state calculations. Spin-orbit coupling was calculated using the state-interacting method as implemented in the Molpro program ${ }^{63,64}$ along with the ECP46MDF basis set. ${ }^{65}$ The $D_{2 \mathrm{~h}}$ symmetry group was used for calculations; irreducible representations (IRs) in the $D_{\infty \mathrm{h}}$ symmetry group were reconstructed from the respective IRs in $D_{2 \mathrm{~h}} D_{2 \mathrm{~h}}$. "Very Tight" optimization criteria were used for optimization; for optimization in the floppy excited state in $\mathrm{Cs}_{2} \mathrm{He}^{+}$, even stricter criteria for solving the EOM-CCSD equations were used, with convergence criteria of $10^{-9}$, $10^{-11}$ and $10^{-10}$ for energy, wavefunction and CCSD and groundstate $Z$-vector iterations, respectively. In all calculations, only 17 electrons in $\mathrm{Cs}_{2}{ }^{+}$were treated explicitly, with the rest described within an effective core pseudopotential (ECP).

Purely dissociative as well as bound excited states are encountered in $\mathrm{Cs}_{2}{ }^{+}$. The absorption spectra of the dissociative states were modeled using the linear reflection principle (LRP) within the harmonic approximation ${ }^{66-68}$ and by the standard reflection principle projecting the vibrational wavefunction (calculated for the $\mathrm{Cs}_{2}{ }^{+}$potential on a grid) onto the excited state potential energy surface. The spectra of the bound states were calculated using the Franck-Condon approximation within the harmonic regime as implemented in the Gaussian software ${ }^{69}$ or directly by calculating the overlap of the ground state vibrational wavefunction in the electronic ground state with both ground and excited vibrational wavefunctions in the electronically excited state.

Gaussian 16 was used for TDDFT and (EOM-)CCSD calculations, Molpro for MRCI and spin-orbit calculations.

\section{Results and discussion}

Let us start with the photodissociation spectrum of $\mathrm{Cs}_{2} \mathrm{He}^{+}$ (Fig. 1a). Upon electronic excitation, the $\mathrm{He}$ atom is lost, and
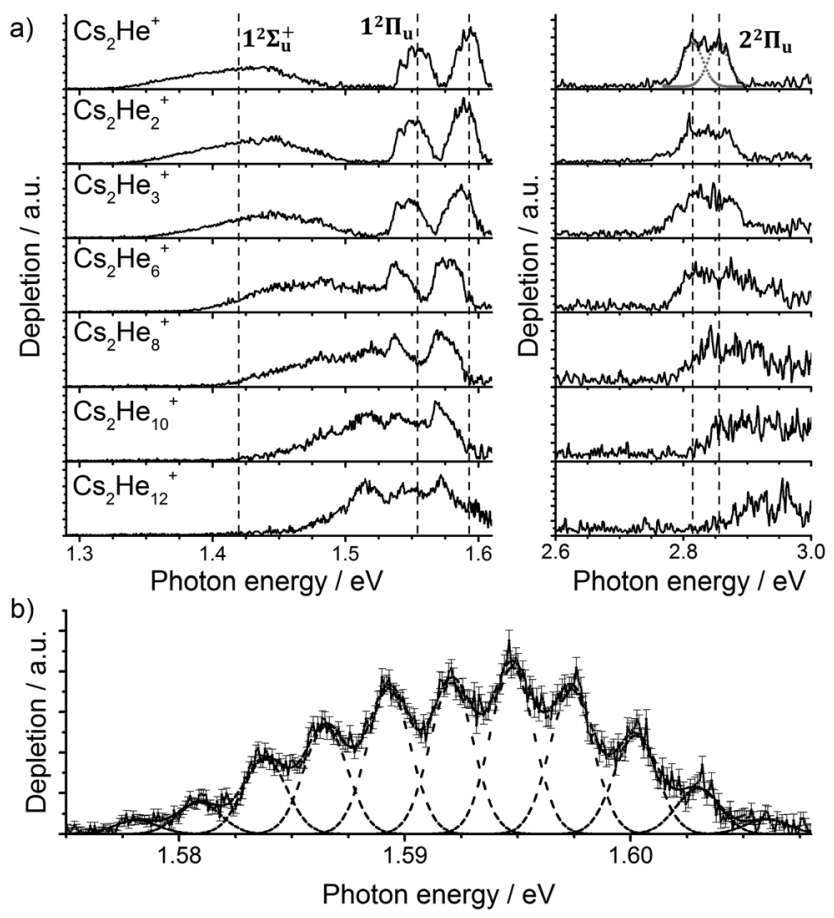

Fig. 1 (a) Photodissociation spectra of $\mathrm{Cs}_{2} \mathrm{He}_{n}^{+}$ions, $n=1,2,3,6,8,10,12$ obtained as a differential spectrum with and without laser irradiation of clusters and scaled with laser power (see ESI $\dagger$ for details). A fit of the peak at $2.8 \mathrm{eV}$ by two Gaussian functions is shown for $n=1$. Band centers for $\mathrm{Cs}_{2} \mathrm{He}^{+}$are marked by vertical lines to guide the eye. Electronic state assignment is based on calculations (Table 1). (b) Multiple Gaussian fit to the vibrational progression of the transition into the $1^{2} \Pi_{\mathrm{u}}$ state in $\mathrm{Cs}_{2} \mathrm{He}^{+}$ and determination of the vibrational spacing. 
Table 1 Experimental and theoretical properties of excited states in $\mathrm{Cs}_{2}{ }^{+}$ and $\mathrm{Cs}_{2} \mathrm{He}^{+}$: energy position $E$ (in $\mathrm{eV}$ ), width $w$ of the Gaussian peak fit $(=2 \sigma)$, experimental splitting $\Delta E_{\text {split, }}$ spin-orbit splitting $\Delta E_{\mathrm{SO}}$ (all in meV). Excitation energies were calculated at the EOM-CCSD/def2QZVPPD level of theory, spectral width was modelled within reflection principle approximation, spin-orbit coupling at the MRCI(1,17)/ECP46MDF level

\begin{tabular}{lllll}
\hline State & & $1^{2} \Sigma_{\mathrm{u}}^{+}$ & $1^{2} \Pi_{\mathrm{u}}$ & $2^{2} \Pi_{\mathrm{u}}$ \\
\hline Experiment, $\mathrm{Cs}_{2} \mathrm{He}^{+}$ & $E$ & 1.41 & $1.55 ; 1.59$ & $2.82 ; 2.86$ \\
& $w$ & 83 & - & $39 ; 30$ \\
& $\Delta E_{\text {split }}$ & - & 39 & 42 \\
Theory, $\mathrm{Cs}_{2}{ }^{+}$ & $E$ & 1.43 & 1.51 & 2.85 \\
& $w$ & 64 & - & 26 \\
Theory, $\mathrm{Cs}_{2} \mathrm{He}^{+}$ & $\Delta E_{\mathrm{SO}}$ & - & 28 & 13 \\
& $E$ & 1.44 & 1.51 & 2.84 \\
& $\Delta E_{\mathrm{SO}}$ & - & 28 & 14
\end{tabular}

the spectrum is recorded following the depletion of the ion signal. There are four clearly observed absorption bands at about 1.4, 1.55, 1.60 and $2.8 \mathrm{eV}$ (Table 1). The band at $2.8 \mathrm{eV}$ is further split, with spacing of about $40 \mathrm{meV}\left(300 \mathrm{~cm}^{-1}\right)$. The band at $1.60 \mathrm{eV}$ has pronounced vibrational structure, with a spacing of $22 \mathrm{~cm}^{-1}$ (Fig. 1b); the broad bands at 1.4 and $2.8 \mathrm{eV}$ are, on the other hand, structureless.

For a more quantitative interpretation and reliable assignment of these features, we performed EOM-CCSD and MRCI calculations and analyzed the contribution of spin-orbit coupling effects. Fig. 2 shows the potential energy curves of $\mathrm{Cs}_{2}{ }^{+}$. The $\mathrm{Cs}_{2}{ }^{+}$ion has $D_{\infty \mathrm{h}}$ symmetry and, when disregarding spin-orbit coupling, its ground electronic state is $1^{2} \Sigma_{g}^{+}$. From this state, the only symmetry allowed transitions are perpendicular and parallel transitions into ${ }^{2} \Pi_{\mathrm{u}}$ and ${ }^{2} \Sigma_{\mathrm{u}}^{+}$states, respectively. Within $3 \mathrm{eV}$, two ${ }^{2} \Sigma_{\mathrm{u}}^{+}$and two ${ }^{2} \Pi_{\mathrm{u}}$ states can be found. Among these states, only the $1^{2} \Pi_{\mathrm{u}}$ state is bound, the others are dissociative. When accounting for spin-orbit effects

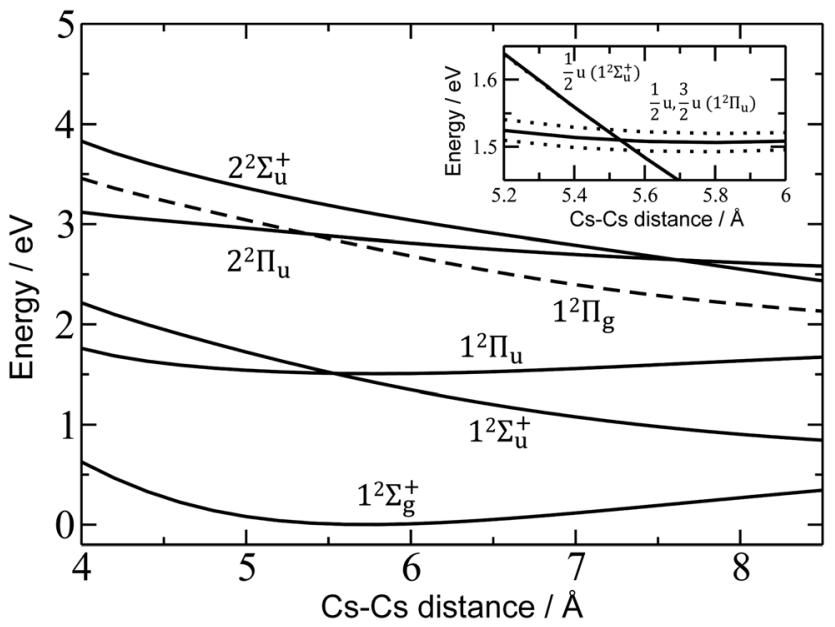

Fig. 2 Potential energy curves in $\mathrm{Cs}_{2}{ }^{+}$calculated at the EOM-CCSD/ def2QZVPPD level. Excited states to which electronic transitions are allowed from the ground state are shown with full lines, forbidden ones with dashed lines. In the inset, the vicinity of the $1^{2} \Pi_{u}$ minimum is shown, along with states including spin-orbit coupling (dotted lines, calculated at the MRCI $(1,17) / E C P 46 M D F$ level of theory). Only selected states are shown. See the ESI† for excluded electronic states and method benchmarking. (see Table S3 in the ESI $\uparrow$ ), the $\Sigma$ states are only slightly affected, with shifts of up to $5 \mathrm{meV}$. However, the doubly-degenerate $\Pi$ states split into two states, separated by $\sim 10-35 \mathrm{meV}\left(80-280 \mathrm{~cm}^{-1}\right)$.

Comparison of the calculations with experiment, summarized in Table 1, allows us to assign the three main bands observed in the experiment as $1^{2} \Sigma_{\mathrm{u}}^{+}, 1^{2} \Pi_{\mathrm{u}}$, and $2^{2} \Pi_{\mathrm{u}}$, reproducing the excitation energy to within $0.1 \mathrm{eV}$. The fourth allowed transition into $2^{2} \Sigma_{\mathrm{u}}^{+}$at $\sim 3.1 \mathrm{eV}$ (see Table S2, ESI $\dagger$ ) is not observed in the experiment due to insufficient laser power in this region. For the dissociative states $1^{2} \Sigma_{\mathrm{u}}^{+}$and $2^{2} \Pi_{\mathrm{u}}$, calculations within the reflection principle approximation also predict a realistic width of the observed bands, see Table 1.

As mentioned above, the $1^{2} \Pi_{\mathrm{u}}$ state is bound and, therefore, the measured photodissociation spectrum reflects its vibrational structure. The $1^{2} \Pi_{u}$ state is calculated to have a very similar

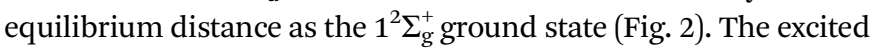
state potential well is less steep, reflected in the calculated harmonic vibrational frequencies of 30 and $20 \mathrm{~cm}^{-1}$ for the $1^{2} \Sigma_{\mathrm{g}}^{+}$and $1^{2} \Pi_{\mathrm{u}}$ state, respectively, for $\mathrm{Cs}_{2}{ }^{+}$(at the (EOM-)CCSD/ def2QZVPPD level). The respective values for $\mathrm{Cs}_{2}{ }^{+} \mathrm{He}$ are 31 and $19 \mathrm{~cm}^{-1}$, with a stronger $\mathrm{Cs}_{2}{ }^{+}-\mathrm{He}$ interaction in the excited states, shortening the Cs-He distance from $4.4 \AA$ in the $1^{2} \Sigma_{g}^{+}$state to $4.0 \AA$ in $1^{2} \Pi_{\mathrm{u}}$. The harmonic $\mathrm{Cs}_{2}{ }^{+} \cdots$ He vibration is located at $30 \mathrm{~cm}^{-1}$. The calculated frequency in the $1^{2} \Pi_{\mathrm{u}}$ state of $19 \mathrm{~cm}^{-1}$ fits well to the measured spacing of $22 \mathrm{~cm}^{-1}$. Note that only the higher $3 / 2 \mathrm{u}\left(1^{2} \Pi_{\mathrm{u}}\right)$ state shows clear vibrational resolution shown in Fig. $1 \mathrm{~b}$. The $1 / 2 \mathrm{u}\left(1^{2} \Pi_{\mathrm{u}}\right)$ state interacts with the repulsive $1 / 2 \mathrm{u}\left(1^{2} \Sigma_{\mathrm{u}}^{+}\right)$state, and the potential energy surface will be deformed due to the avoided crossing of the states. From a computational perspective, the equilibrium distance in the $1^{2} \Pi_{u}$ state depends heavily on the choice of the basis set (Fig. S4, ESI†). As a direct consequence, our attempts to reproduce the experimental spectrum shown in Fig. 1b using Franck-Condon simulations failed to grasp correctly the relative intensity of the contributing peaks (see the ESI $\dagger$ for details).

Finally, the calculated weak spin-orbit splitting of the ${ }^{2} \Pi_{u}$ states results into two states $1 / 2 \mathrm{u}$ and $3 / 2 \mathrm{u}$ with a different $\Omega$ value following Hund's rule 3. The splitting is well reproduced for $1^{2} \Pi_{u}$ (see also inset in Fig. 2). For $2^{2} \Pi_{u}$, the calculated spinorbit splitting is significantly smaller than the experimentally observed splitting. These observations can be rationalized if one takes into account the additional splitting induced by the asymmetric distribution of the $\mathrm{He}$ atom relative to the singly occupied molecular orbital (SOMO) in the excited state. Fig. 3 illustrates how the energy of the several electronic states in $\mathrm{Cs}_{2} \mathrm{He}^{+}$depends on the position of the He atom in $\mathrm{Cs}_{2} \mathrm{He}^{+}$. For this calculation, the Cs-Cs-He angle is fixed, and the Cs-Cs and Cs-He distances are optimized in the electronic ground state, $1^{2} \Sigma_{\mathrm{g}}^{+}$. From $180^{\circ}$ to $150^{\circ}$, the ground state energy hardly changes, i.e. the $\mathrm{He}$ atom moves more or less freely in this range of Cs-Cs-He angles. The repulsive $1^{2} \Sigma_{\mathrm{u}}^{+}$state reacts more sensitively to the position of the $\mathrm{He}$ atom, and this effect contributes to the broadening of the absorption band, which is $19 \mathrm{meV}$ larger than expected from the linear reflection principle approximation, Table 1 . For the $1^{2} \Pi_{u}$ and $2^{2} \Pi_{u}$ states, the degeneracy of the two contributing $\pi$ orbitals (see Fig. 4 ) is 


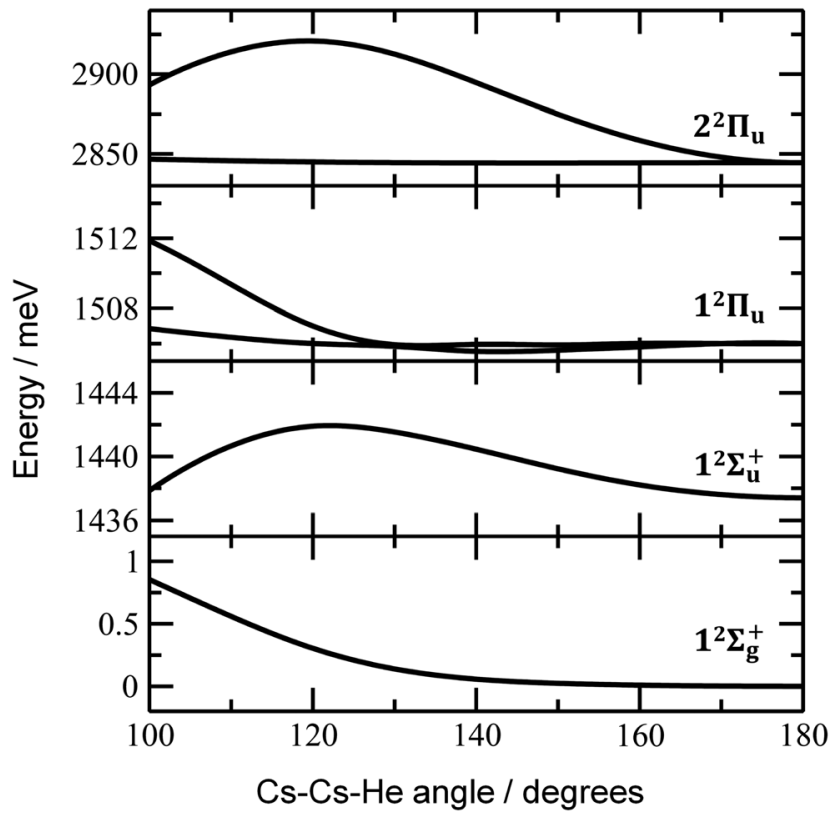

Fig. 3 Dependence of ground state and excited state energies in $\mathrm{Cs}_{2} \mathrm{He}^{+}$ on the Cs-Cs-He angle. Calculated at the EOM-CCSD/def2QZVPPD level of theory with ground-state structures optimized at the CCSD/ def2QZVP(Cs), def2TZVP(He) level with a constrained Cs-Cs-He angle. Spin-orbit effects were neglected.

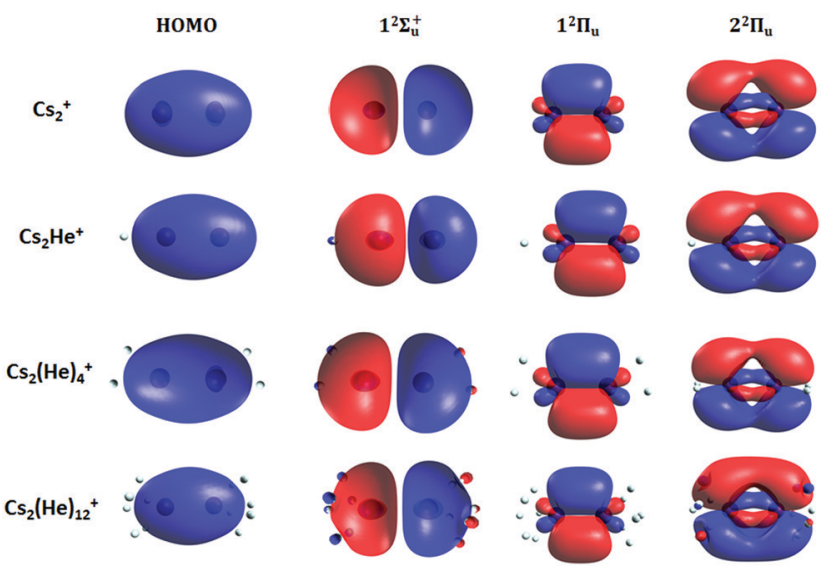

Fig. 4 Highest occupied molecular orbital (HOMO) and natural transition orbitals for $\mathrm{Cs}_{2} \mathrm{He}_{n}^{+}$ions calculated at the CAM-B3LYP/def2QZVPPD level of theory. A low iso value (0.005) was chosen so that orbital deformation induced by helium is clearly visible.

lifted by the presence of the He atom, which leads to an additional splitting. For $1^{2} \Pi_{u}$, this effect is negligible down to $150^{\circ} \mathrm{Cs}-\mathrm{Cs}-\mathrm{He}$ angle, because the He atom does occupy the same space as the singly occupied $\pi$ orbitals. In contrast, the SOMO of the $2^{2} \Pi_{u}$ state is much more diffuse, and in one of the two orientations exhibits significant overlap with the position of the He atom. The splitting of the two orientations of the SOMO in the $2^{2} \Pi_{\mathrm{u}}$ state amounts to $31 \mathrm{meV}$ at $150^{\circ}$, which constitutes the dominant contribution to the experimentally observed splitting. For larger clusters, the same effect is responsible for the significant broadening of the $2^{2} \Pi_{\mathrm{u}}$ band.
Let us now concentrate on the shift of the spectra induced by solvation, i.e. the He matrix shift. As shown in Fig. 1a and Table 2, pronounced shifts to higher energies are recorded for the $1^{2} \Sigma_{\mathrm{u}}^{+}$and $2^{2} \Pi_{\mathrm{u}}$ states, reaching values of more than $0.1 \mathrm{eV}$ already for $\mathrm{Cs}_{2} \mathrm{He}_{10}{ }^{+}$. Note also that the shift in the $1^{2} \Sigma_{\mathrm{u}}^{+}$state exceeds the He binding energy. In the $n=1-10$ range, the energy of the $1^{2} \Sigma_{\mathrm{u}}^{+}$state shifts linearly with solvation by $12 \mathrm{meV}$ $\left(100 \mathrm{~cm}^{-1}\right)$ per helium atom. The $2^{2} \Pi_{\mathrm{u}}$ band at $\sim 2.8 \mathrm{eV}$ seems to shift slightly non-linearly, its structure is however not well resolved in the experiment for larger ions. The $1^{2} \Pi_{\mathrm{u}}$ state at $1.6 \mathrm{eV}$, on the other hand, is shifted only by $\sim 20 \mathrm{meV}\left(160 \mathrm{~cm}^{-1}\right)$ to lower energies within $n=1-12$ (Table 2).

The shifts can be directly connected to the interplay between the position of $\mathrm{He}$ atoms around the $\mathrm{Cs}_{2}{ }^{+}$ion and the character of the respective excited state. Our calculations show that $\mathrm{Cs}_{2}{ }^{+}$ is preferentially solvated by $\mathrm{He}$ atoms on its ends, along the Cs-Cs axis for the smallest ions (see Fig. 4). For $\mathrm{Cs}_{2} \mathrm{He}^{+}$and $\mathrm{Cs}_{2} \mathrm{He}_{2}{ }^{+}$, the most stable configuration is linear, but deformation from linearity down to a Cs-Cs-He angle of $150^{\circ}$ requires very little energy, see Fig. 3; in $\mathrm{Cs}_{2} \mathrm{He}_{12}{ }^{+}$, conformations with six and five/seven He atoms on each end are the most stable ones (Table S1, ESI $\dagger$ ). As expected, the binding energy of helium atoms is low, within 2.4-3.1 meV He $\mathrm{He}^{-1}$ for up to 12 helium atoms. In addition to the shifts, the presence of a large variety of almost isoenergetic isomers leads to significant broadening of the spectra upon solvation (see Fig. S1, ESI†).

Due to Pauli repulsion, the presence of He atoms deforms the orbitals of both ground and excited electronic states that extend along the Cs-Cs axis, shifting them to higher energy. This is the case for the HOMO orbital as well as the orbitals corresponding to excitations into the $1^{2} \Sigma_{\mathrm{u}}^{+}$and $2^{2} \Pi_{\mathrm{u}}$ states (Fig. 4). The diffuse orbitals in the excited state are deformed more than the HOMO orbital, shifting the excitation energy considerably to higher values (Fig. 1a). The $\pi$ orbital corresponding to the excitation into the $1^{2} \Pi_{\mathrm{u}}$ state at $1.6 \mathrm{eV}$ represents a different case. Here, the surrounding helium atoms do not approach the orbital and the shift to lower energies reflects the slight destabilization of the HOMO orbital. Our calculations can reproduce the shifts for excitations into $1^{2} \Sigma_{\mathrm{u}}^{+}$and underestimate those observed for $1^{2} \Pi_{u}$ (Table 2); for $n=12$, the

Table 2 Experimental and calculated energy shift of excited states upon solvation (in meV) with respect to $\mathrm{Cs}_{2} \mathrm{He}^{+}$. See Fig. S12 for experimental fits. Electronic states are correlated to $1^{2} \Sigma_{\mathrm{u}}^{+}$and $1^{2} \Pi_{\mathrm{u}}$ states in $\mathrm{Cs}_{2}{ }^{+}$. Vertical transitions were calculated at the EOM-CCSD/def2QZVPPD//CCSD/ def2QZVP(Cs), def2TZVP(He) level of theory. For the $1^{2} \Pi_{u}$ state, the average calculated value of the two SOMO orientations is given. Isomers with equal distribution of $\mathrm{He}$ atoms to each $\mathrm{Cs}_{2}{ }^{+}$end are chosen for the calculation

\begin{tabular}{llllll}
\hline & \multicolumn{2}{l}{ Experiment } & & \multicolumn{2}{c}{ Theory } \\
\cline { 2 - 3 } \cline { 5 - 6 }$n_{\mathrm{He}}$ & $1^{2} \Sigma_{\mathrm{u}}^{+}$ & $1^{2} \Pi_{\mathrm{u}}$ & & $1^{2} \Sigma_{\mathrm{u}}^{+}$ & $1^{2} \Pi_{\mathrm{u}}$ \\
\hline 2 & 11 & $-3.4 ;-3.7$ & 6 & -0.8 \\
3 & 23 & $-6.1 ;-6.6$ & 15 & -2.1 \\
6 & 59 & $-13.3 ;-15.2$ & 41 & -6.1 \\
10 & 98 & $-9.2 ;-19.3$ & 96 & -12.0 \\
12 & 124 & $-18.5 ;-41.0$ & 109 & -14.2
\end{tabular}


sudden increase observed in the $1^{2} \Pi_{u}$ experimental shift might be induced by the fitting procedure (see Fig. S12, ESI $\dagger$ ) or appearance of new isomers. This is most likely caused by the neglect of He delocalization around the $\mathrm{Cs}_{2}{ }^{+}$core due to zeropoint motion and the presence of multiple isomers.

\section{Conclusions}

In contrast to photodissociation of bare $\mathrm{Cs}_{2}{ }^{+}$, helium tagging gives experimental access to the $3 / 2 \mathrm{u}$ component of the $1^{2} \Pi_{u}$ state, which features a pronounced vibrational progression with a Cs-Cs frequency of $22 \mathrm{~cm}^{-1}$ in the excited state. According to quantum chemical calculations, He considerably changes the spectrum already for the $\mathrm{Cs}_{2} \mathrm{He}^{+}$ion, inducing splitting of bands due to both solvation and dynamic effects. When more helium atoms are added, shifts exceeding $0.1 \mathrm{eV}$, i.e. almost $1000 \mathrm{~cm}^{-1}$, are induced, and the $2^{2} \Pi_{u}$ significantly broadens. The magnitude and even direction of the shift are extremely sensitive to both the nature of the electronic transition as well as the position of the solvating atoms. Our calculations show that the shifts are particularly pronounced if He occupies a position in the electronic ground state that carries large electron density in the excited state. Despite its extremely weak interaction, He is capable of inducing significant matrix shifts in electronic excitation spectra, due to Pauli repulsion, as already pointed out before. ${ }^{70,71}$ This effect is used to derive information both on the shape of excited state molecular orbitals as well as solvation patterns.

\section{Conflicts of interest}

There are no conflicts to declare.

\section{Acknowledgements}

This work was supported by the Austrian Science Fund FWF, project numbers W1259 (DK-ALM), P29174 and P31149, and the Swedish Research Council, contract number 2016-06625. The tunable OPO system is part of the Innsbruck Laser Core Facility, financed by the Austrian Federal Ministry of Science, Research and Economy. The computational results presented have been achieved using the HPC infrastructure LEO of the University of Innsbruck.

\section{References}

1 M. Förstel, W. Schewe and O. Dopfer, Angew. Chem., Int. Ed., 2019, 58, 3356-3360.

2 J. Jankunas and A. Osterwalder, Annu. Rev. Phys. Chem., 2015, 66, 241-262, and references therein.

3 D. H. Levy, Annu. Rev. Phys. Chem., 1980, 31, 197-225, and references therein.

4 V. E. Bondybey, A. M. Smith and J. Agreiter, Chem. Rev., 1996, 96, 2113-2134, and references therein.

5 V. A. Apkarian and N. Schwentner, Chem. Rev., 1999, 99, 1481-1514, and references therein.
6 J. P. Toennies and A. F. Vilesov, Angew. Chem., Int. Ed., 2004, 43, 2622-2648, and references therein.

7 A. Mauracher, O. Echt, A. M. Ellis, S. Yang, D. K. Bohme, J. Postler, A. Kaiser, S. Denifl and P. Scheier, Phys. Rep., 2018, 751, 1-90, and references therein.

8 E. J. Bieske and O. Dopfer, Chem. Rev., 2000, 100, 3963-3998, and references therein.

9 J. A. Fournier, C. J. Johnson, C. T. Wolke, G. H. Weddle, A. B. Wolk and M. A. Johnson, Science, 2014, 344, 1009-1012.

10 E. K. Campbell, M. Holz, D. Gerlich and J. P. Maier, Nature, 2015, 523, 322-323.

11 E. K. Campbell, M. Holz and J. P. Maier, Astrophys. J., Lett., 2016, 826, L4.

12 E. K. Campbell, M. Holz, J. P. Maier, D. Gerlich, G. A. H. Walker and D. Bohlender, Astrophys. J., 2016, 822, 17.

13 J. P. Maier and E. K. Campbell, Philos. Trans. R. Soc., A, 2016, 374, 20150316.

14 J. Roithova, A. Gray, E. Andris, J. Jasik and D. Gerlich, Acc. Chem. Res., 2016, 49, 223-230.

15 O. Asvany, S. Brünken, L. Kluge and S. Schlemmer, Appl. Phys. B: Lasers Opt., 2014, 114, 203-211.

16 O. Asvany, K. M. T. Yamada, S. Brünken, A. Potapov and S. Schlemmer, Science, 2015, 347, 1346-1349.

17 O. Lakhmanskaya, M. Simpson, S. Murauer, M. Notzold, E. Endres, V. Kokoouline and R. Wester, Phys. Rev. Lett., 2018, 120, 253003.

18 R. L. Wong, K. Paech and E. R. Williams, Int. J. Mass Spectrom., 2004, 232, 59-66.

19 O. P. Balaj, C. B. Berg, S. J. Reitmeier, V. E. Bondybey and M. K. Beyer, Int. J. Mass Spectrom., 2009, 279, 5-9.

20 S. Dillinger, M. P. Klein, A. Steiner, D. C. McDonald, M. A. Duncan, M. M. Kappes and G. Niedner-Schatteburg, J. Phys. Chem. Lett., 2018, 9, 914-918.

21 R. D. Thomas, H. T. Schmidt, M. Gatchell, S. Rosen, P. Reinhed, P. Lofgren, L. Brannholm, M. Blom, M. Bjorkhage, E. Backstrom, J. D. Alexander, S. Leontein, D. Hanstorp, H. Zettergren, M. Kaminska, R. Nascimento, L. Liljeby, A. Kallberg, A. Simonsson, F. Hellberg, S. Mannervik, M. Larsson, W. D. Geppert, K. G. Rensfelt, A. Paal, M. Masuda, P. Hallden, G. Andler, M. H. Stockett, T. Chen, G. Kallersjo, J. Weimer, K. Hansen, H. Hartman and H. Cederquist, EPJ Web Conf., 2015, 84, 01004.

22 M. Gatchell, H. T. Schmidt, R. D. Thomas, S. Rosen, P. Reinhed, P. Lofgren, L. Brannholm, M. Blom, M. Bjorkhage, E. Backstrom, J. D. Alexander, S. Leontein, D. Hanstorp, H. Zettergren, L. Liljeby, A. Kallberg, A. Simonsson, F. Hellberg, S. Mannervik, M. Larsson, W. D. Geppert, K. G. Rensfelt, H. Danared, A. Paal, M. Masuda, P. Hallden, G. Andler, M. H. Stockett, T. Chen, G. Kallersjo, J. Weimer, K. Hansen, H. Hartman and H. Cederquist, J. Phys.: Conf. Ser., 2014, 488, 012040.

23 R. D. Thomas, H. T. Schmidt, J. Gurell, N. Haag, A. I. S. Holm, H. A. B. Johansson, G. Kallersjo, M. Larsson, S. Mannervik, D. Misra, A. Orban, P. Reinhed, K. G. Rensfelt, S. Rosen, F. Seitz, J. Weimer, H. Zettergren, G. Andler, H. Danared, S. Das, L. Liljeby, M. Bjorkhage, M. Blom, 
L. Brannholm, P. Hallden, F. Hellberg, A. Kallberg, S. Leontein, P. Lofgren, B. Malm, A. Paal, A. Simonsson and H. Cederquist, J. Phys.: Conf. Ser., 2011, 300, 012011.

24 R. von Hahn, A. Becker, F. Berg, K. Blaum, C. Breitenfeldt, H. Fadil, F. Fellenberger, M. Froese, S. George, J. Gock, M. Grieser, F. Grussie, E. A. Guerin, O. Heber, P. Herwig, J. Karthein, C. Krantz, H. Kreckel, M. Lange, F. Laux, S. Lohmann, S. Menk, C. Meyer, P. M. Mishra, O. Novotny, A. P. O'Connor, D. A. Orlov, M. L. Rappaport, R. Repnow, S. Saurabh, S. Schippers, C. D. Schroter, D. Schwalm, L. Schweikhard, T. Sieber, A. Shornikov, K. Spruck, S. S. Kumar, J. Ullich, X. Urbain, S. Vogel, P. Wilhelm, A. Wolf and D. Zajfman, Rev. Sci. Instrum., 2016, 87, 063115.

25 Y. Nakano, Y. Enomoto, T. Masunaga, S. Menk, P. Bertier and T. Azuma, Rev. Sci. Instrum., 2017, 88, 033110.

26 G. Berden, R. Peeters and G. Meijer, Int. Rev. Phys. Chem., 2000, 19, 565-607, and references therein.

27 T. D. Fridgen, Mass Spectrom. Rev., 2009, 28, 586-607, and references therein.

28 N. C. Polfer and J. Oomens, Mass Spectrom. Rev., 2009, 28, 468-494, and references therein.

29 M. Straka, E. Andris, J. Vicha, A. Ruzicka, J. Roithova and L. Rulisek, Angew. Chem., Int. Ed., 2019, 58, 2011-2016.

30 G. T. Pullen, P. R. Franke, Y. P. Lee and G. E. Douberly, J. Mol. Spectrosc., 2018, 354, 7-14.

31 M. Kuhn, M. Renzler, J. Postler, S. Ralser, S. Spieler, M. Simpson, H. Linnartz, A. G. G. M. Tielens, J. Cami, A. Mauracher, Y. Wang, M. Alcamí, F. Martín, M. K. Beyer, R. Wester, A. Lindinger and P. Scheier, Nat. Commun., 2016, 7, 13550.

32 S. Spieler, M. Kuhn, J. Postler, M. Simpson, R. Wester, P. Scheier, W. Ubachs, X. Bacalla, J. Bouwman and H. Linnartz, Astrophys. J., 2017, 846, 168.

33 A. Kaiser, J. Postler, M. Oncak, M. Kuhn, M. Renzler, S. Spieler, M. Simpson, M. Gatchell, M. K. Beyer, R. Wester, F. A. Gianturco, P. Scheier, F. Calvo and E. Yurtsever, J. Phys. Chem. Lett., 2018, 9, 1237-1242.

34 F. Stienkemeier, J. Higgins, C. Callegari, S. I. Kanorsky, W. E. Ernst and G. Scoles, Z. Phys. D: At., Mol. Clusters, 1996, 38, 253-263.

35 J. Higgins, C. Callegari, J. Reho, F. Stienkemeier, W. E. Ernst, M. Gutowski and G. Scoles, J. Phys. Chem. A, 1998, 102, 4952-4965.

36 J. H. Reho, J. Higgins, M. Nooijen, K. K. Lehmann, G. Scoles and M. Gutowski, J. Chem. Phys., 2001, 115, 10265-10274.

37 G. E. Douberly and R. E. Miller, J. Phys. Chem. A, 2007, 111, 7292-7302.

38 J. Tiggesbäumker and F. Stienkemeier, Phys. Chem. Chem. Phys., 2007, 9, 4748-4770.

39 M. Theisen, F. Lackner and W. E. Ernst, J. Chem. Phys., 2011, 135, 074306.

40 M. Renzler, M. Daxner, L. Kranabetter, A. Kaiser, A. W. Hauser, W. E. Ernst, A. Lindinger, R. Zillich, P. Scheier and A. M. Ellis, J. Chem. Phys., 2016, 145, 181101.

41 E. Cheng, M. W. Cole, W. F. Saam and J. Treiner, Phys. Rev. Lett., 1991, 67, 1007-1010.
42 F. Ancilotto, E. Cheng, M. W. Cole and F. Toigo, Z. Phys. B: Condens. Matter, 1995, 98, 323-329.

43 F. Ancilotto, A. M. Sartori and F. Toigo, Phys. Rev. B: Condens. Matter Mater. Phys., 1998, 58, 5085-5092.

44 A. Nakayama and K. Yamashita, J. Chem. Phys., 2000, 112, 10966-10975.

45 A. Nakayama and K. Yamashita, AIP Conf. Proc., 2001, 559, 265-272.

46 C. Stark and V. V. Kresin, Phys. Rev. B: Condens. Matter Mater. Phys., 2010, 81, 085401.

47 L. An der Lan, P. Bartl, C. Leidlmair, H. Schöbel, R. Jochum, S. Denifl, T. D. Märk, A. M. Ellis and P. Scheier, J. Chem. Phys., 2011, 135, 044309.

48 L. An der Lan, P. Bartl, C. Leidlmair, H. Schöbel, S. Denifl, T. D. Märk, A. M. Ellis and P. Scheier, Phys. Rev. B: Condens. Matter Mater. Phys., 2012, 85, 115414.

49 L. Chen, J. Zhang, W. M. Freund and W. Kong, J. Chem. Phys., 2015, 143, 074201.

50 M. Rastogi, C. Leidlmair, L. An der Lan, J. O. de Zarate, R. P. de Tudela, M. Bartolomei, M. I. Hernandez, J. CamposMartinez, T. Gonzalez-Lezana, J. Hernandez-Rojas, J. Breton, P. Scheier and M. Gatchell, Phys. Chem. Chem. Phys., 2018, 20, 25569-25576.

51 L. Kranabetter, M. Goulart, A. Aleem, T. Kurzthaler, M. Kuhn, E. Barwa, M. Renzler, L. Grubwieser, M. Schwärzler, A. Kaiser, P. Scheier and O. Echt, J. Phys. Chem. C, 2017, 121, 10887-10892.

52 R. Pérez de Tudela, P. Martini, M. Goulart, P. Scheier, F. Pirani, J. Hernandez-Rojas, J. Breton, J. Ortiz de Zárate, M. Bartolomei, T. Gonzalez-Lezana, M. I. Hernandez, J. Campos-Martinez and P. Villarreal, J. Chem. Phys., 2019, 150, 154304.

53 H. Kohguchi, P. Jusko, K. M. T. Yamada, S. Schlemmer and O. Asvany, J. Chem. Phys., 2018, 148, 144303.

54 H. Helm, P. C. Cosby and D. L. Huestis, J. Chem. Phys., 1983, 78, 6451-6454.

55 H. Helm and R. Möller, Phys. Rev. A: At., Mol., Opt. Phys., 1983, 27, 2493-2502.

56 M. Aymar, S. Azizi and O. Dulieu, J. Phys. B: At., Mol. Opt. Phys., 2003, 36, 4799-4812.

57 A. Jraij, A. R. Allouche, M. Korek and M. Aubert-Frecon, Chem. Phys., 2005, 310, 145-151.

58 H. Silberbach, P. Schwerdtfeger, H. Stoll and H. Preuss, J. Phys. B: At., Mol. Opt. Phys., 1986, 19, 501-510.

59 A. Scheidemann, B. Schilling and J. P. Toennies, J. Phys. Chem., 1993, 97, 2128-2138.

60 R. Fröchtenicht, U. Henne, J. P. Toennies, A. Ding, M. Fieber-Erdmann and T. Drewello, J. Chem. Phys., 1996, 104, 2548-2556.

61 A. C. LaForge, M. Shcherbinin, F. Stienkemeier, R. Richter, R. Moshammer, T. Pfeifer and M. Mudrich, Nat. Phys., 2019, 15, 247-250.

62 M. Gatchell, P. Martini, F. Laimer, M. Goulart, F. Calvo and P. Scheier, Faraday Discuss., 2019, 217, 276-289.

63 H.-J. Werner, P. J. Knowles, G. Knizia, F. R. Manby, M. Schütz, P. Celani, T. Korona, R. Lindh, A. Mitrushenkov, G. Rauhut, K. R. Shamasundar, T. B. Adler, R. D. Amos, A. Bernhardsson, 
A. Berning, D. L. Cooper, M. J. O. Deegan, A. J. Dobbyn, F. Eckert, E. Goll, C. Hampel, A. Hesselmann, G. Hetzer, T. Hrenar, G. Jansen, C. Köppl, Y. Liu, A. W. Lloyd, R. A. Mata, A. J. May, S. J. McNicholas, W. Meyer, M. E. Mura, A. Nicklass, D. P. O’Neill, P. Palmieri, D. Peng, K. Pflüger, R. Pitzer, M. Reiher, T. Shiozaki, H. Stoll, A. J. Stone, R. Tarroni, T. Thorsteinsson and M. Wang, MOLPRO, version 2012.1, a package of ab initio programs, 2012.

64 H. J. Werner, P. J. Knowles, G. Knizia, F. R. Manby and M. Schutz, Wiley Interdiscip. Rev.: Comput. Mol. Sci., 2012, 2, 242-253.

65 I. S. Lim, P. Schwerdtfeger, B. Metz and H. Stoll, J. Chem. Phys., 2005, 122, 104103.

66 S. Y. Lee, R. C. Brown and E. J. Heller, J. Phys. Chem., 1983, 87, 2045-2053.

67 M. K. Prakash, J. D. Weibel and R. A. Marcus, J. Geophys. Res.: Atmos., 2005, 110, D21315.

68 M. Oncak, L. Sistik and P. Slavicek, J. Chem. Phys., 2010, 133, 174303.

69 M. J. Frisch, G. W. Trucks, H. B. Schlegel, G. E. Scuseria, M. A. Robb, J. R. Cheeseman, G. Scalmani, V. Barone,
G. A. Petersson, H. Nakatsuji, X. Li, M. Caricato, A. V. Marenich, J. Bloino, B. G. Janesko, R. Gomperts, B. Mennucci, H. P. Hratchian, J. V. Ortiz, A. F. Izmaylov, J. L. Sonnenberg, D. Williams-Young, F. Ding, F. Lipparini, F. Egidi, J. Goings, B. Peng, A. Petrone, T. Henderson, D. Ranasinghe, V. G. Zakrzewski, J. Gao, N. Rega, G. Zheng, W. Liang, M. Hada, M. Ehara, K. Toyota, R. Fukuda, J. Hasegawa, M. Ishida, T. Nakajima, Y. Honda, O. Kitao, H. Nakai, T. Vreven, K. Throssell, J. A. Montgomery, Jr., J. E. Peralta, F. Ogliaro, M. J. Bearpark, J. J. Heyd, E. N. Brothers, K. N. Kudin, V. N. Staroverov, T. A. Keith, R. Kobayashi, J. Normand, K. Raghavachari, A. P. Rendell, J. C. Burant, S. S. Iyengar, J. Tomasi, M. Cossi, J. M. Millam, M. Klene, C. Adamo, R. Cammi, J. W. Ochterski, R. L. Martin, K. Morokuma, O. Farkas, J. B. Foresman and D. J. Fox, Gaussian 16, Revision A.03, Gaussian, Inc., Wallingford CT, 2016.

70 M. Ratschek, J. V. Pototschnig, A. W. Hauser and W. E. Ernst, J. Phys. Chem. A, 2014, 118, 6622-6631.

71 T. J. O. P. C. A. Messner, A. Schiffmann, J. V. Pototschnig, M. Lasserus, M. Schnedlitz, F. Lackner and W. E. Ernst, J. Chem. Phys., 2018, 149, 024305. 